Найзабеков А. Б. Лежнев С. Н. Панин Е. А.

\title{
ИССЛЕДОВАНИЕ ВЛИЯНИЯ ОСНОВНЫХ ПАРАМЕТРОВ ПРОЦЕССА «ПРОКАТКА-РКУП» НА НАПРЯЖЕННО-ДЕФОРМИРОВАННОЕ СОСТОЯНИЕ И ЭВОЛЮЦИЮ МИКРОСТРУКТУРЫ С ИСПОЛЬЗОВАНИЕМ КОМПЬЮТЕРНОГО МОДЕЛИРОВАНИЯ
}

Создание новых конструкционных материалов с новыми уникальными свойствами металла остается одной из актуальных задач современного материаловедения. На практике можно улучшить свойства существующих конструкционных материалов путем измельчения их структуры до ультрамелкозернистого состояния. На данный момент существует несколько способов получения такой структуры и одним из таких методов является метод интенсивного пластического деформирования (ИПД), реализуемый во всем объеме деформируемой заготовки. В последние годы наметилась тенденция к развитию так называемых «совмещенных» процессов формообразования металлов, которые представляют собой сочетание двух и более традиционных деформационных процессов [1-3]. Основной особенностью совмещенных процессов обработки металлов давлением является то, что зачастую их реализация снижает или полностью устраняет недостатки обычных процессов, входящих в состав совмещенного процесса.

Одним из таких процессов является совмещенный процесс деформирования «прокатка-РКУП» с использованием ступенчатой матрицы и двух пар валков с гладкой бочкой [4] или двух пар калиброванных валков [5]. Этот процесс позволяет получать прямоугольные заготовки с ультрамелкозернистой структурой и повышенными механическими свойствами.

На современном этапе развития науки и техники моделирование различных деформационных процессов в металлообработке имеет большое значение. Во-первых, моделирование позволяет исследователю заглянуть «внутрь» процесса, оценить возникающие напряжения и деформации, спрогнозировать появление дефектов. Во-вторых, это позволяет определить оптимальные параметры инструмента и заготовки для лучшей реализации процесса. Современные программные системы моделирования дают широкие возможности для работы, они позволяют моделировать практически любой процесс, избегая дорогостоящих экспериментов. Поэтому моделирование различных деформационных процессов при обработке металлов давлением является важной задачей.

В ходе теоретических исследований совмещенного процесса «прокатка-РКУП» [6-7] было выявлено, что для осуществления этого процесса необходимо преодолеть силы противодавления, возникающие в матрице. Это может быть достигнуто за счет увеличения усилий, возникающих в зоне деформации при прокатке, величина которых может изменяться путем изменения радиуса валков и абсолютного обжатия при прокатке. Однако при моделировании необходимо также учитывать влияние различных технологических факторов, таких как температура заготовки, от величины которой зависит сопротивление деформации; коэффициенты трения в валках и матрице, а также некоторые другие геометрические факторы, такие как длина каналов в матрице [7]. Таким образом, данная работа посвящена исследованию влияния основных параметров процесса «прокатка-РКУП» на напряженно-деформированное состояние и эволюцию микроструктуры с помощью компьютерного моделирования.

Исследование напряженного состояния является одним из основных этапов разработки нового технологического процесса, поскольку позволяет изучить распределение напряжений в заготовке, а также выявить те участки, которые более склонны к образованию дефектов вследствие возникновения высоких растягивающих напряжений. Это дает возможность 
внести необходимые корректировки для снижения интенсивности действия растягивающих напряжений и предотвращения образования дефектов. На данном этапе работы был проведен анализ напряженного состояния совмещенного процесса «прокатка-РКУП» с использованием калиброванных валков. Для исследования влияния геометрических и технологических параметров на напряженное состояние совмещенного процесса «прокатка-РКУП» в качестве базовой модели использовалась ранее рассмотренная модель для получения заготовок сечением $20 \times 20$ мм [7]: диаметр 1-ой пары валков - 200 мм; диаметр 2-ой пары валков - 220 мм; коэффициент трения в валках - 0,5; коэффициент трения в матрице - 0,1; длина 1-го и 3-го каналов в матрице - 100 мм; длина 2-го канала в матрице - 20 мм, угол стыка каналов - 140; абсолютное обжатие в 1-ой паре валков - 5 мм.

Было решено проанализировать следующий этап деформации: заготовка прокатывается в первой паре валков, перемещается по каналам матрицы, а затем прокатывается во второй паре валков. На этом этапе деформации существует возможность изучения напряженного состояния в матрице и в обеих парах валков. Получены следующие результаты исследования напряженного состояния (рис. 1).

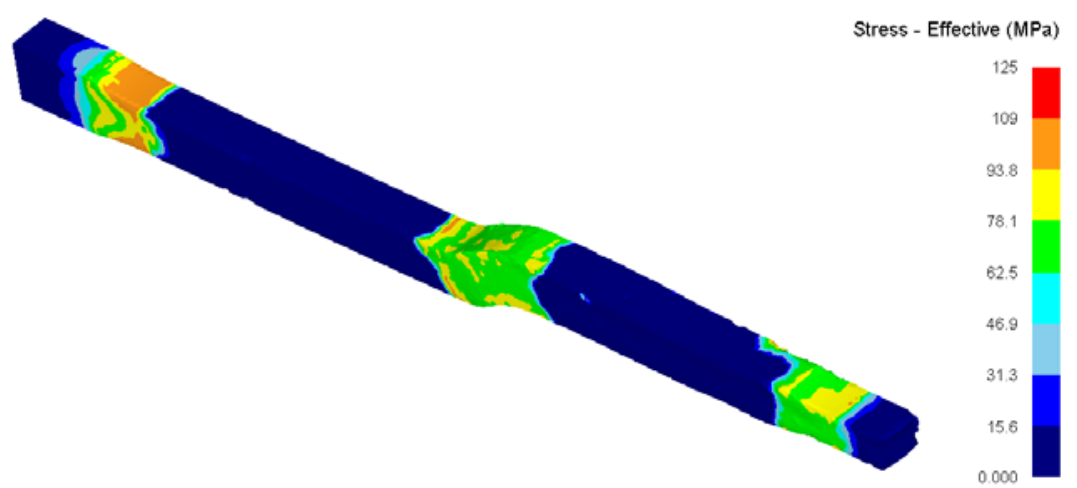

Рис. 1. Эквивалентное напряжение

Рассматривая эквивалентное напряжение, следует понимать, что этот параметр не показывает, какой тип напряжения действует в конкретной точке (растяжение или сжатие). Как радикальное выражение, его значение всегда положительно. Он показывает интенсивность напряжения, то есть напряжение в этой точке или нет. Его величина характеризует среднее значение всех напряжений, действующих в определенной точке. В первой зоне деформации при прокатке в первой паре валков напряжение охватывает всю зону деформации. В зонах контакта металла с валками его значение достигает 105 МПа, на свободных участках значение этого параметра составляет около 75-85 МПа. Во второй зоне деформации, когда заготовка проходит через каналы матрицы, напряжение охватывает широкую область, соединяющую два соединения каналов. За счет действия сдвиговых деформаций величина напряжений в этой зоне деформации снижается до 75-80 МПа. В третьей зоне деформации при прокатке во второй паре валков создаваемые напряжения достигают величины 70-75 МПа в центральных слоях заготовки и 90-95 МПа в поверхностных слоях.

Для исследования деформационного состояния используется показатель интенсивности деформации - эквивалентная деформация, которая включает в себя основные компоненты тензора. Этот параметр позволяет оценить степень деформации, которая является кумулятивным параметром. При исследовании деформированного состояния необходимо не только обеспечить наивысший уровень эквивалентной деформации, необходимый для формирования ультрамелкой структуры, но и равномерное распределение этого параметра по поперечному сечению заготовки.

В первой зоне деформации эквивалентная деформация имеет значение до 0,4 в поверхностных слоях (рис. 2, а); в центральных слоях значение этого параметра равно 0,3 (рис. 2, б), то есть разница значений эквивалентной деформации достигает $33 \%$. Во второй зоне деформации происходит значительное увеличение этого параметра за счет осуществления сдвиговой 
деформации при перемещении заготовки по каналам матрицы. Величина эквивалентной деформации после прохождения первого стыка достигает 0,75 в поверхностных слоях и 0,65 в центральных слоях - здесь разница значений эквивалентной деформации достигает $15 \%$. После прохождения второго пересечения значение эквивалентной деформации достигает 1,0 в поверхностных слоях и 0,9 в центральных слоях, то есть разность значений эквивалентной деформации достигает $11 \%$. Таким образом, разница значений этого параметра после прохождения по каналам матрицы уменьшилась на 22 \%. В третьей зоне деформации при прокатке во второй паре валков величина эквивалентной деформации достигает 1,2 в поверхностных слоях и 1,0 в центральных слоях, то есть разница увеличивается до 20 \%.

a
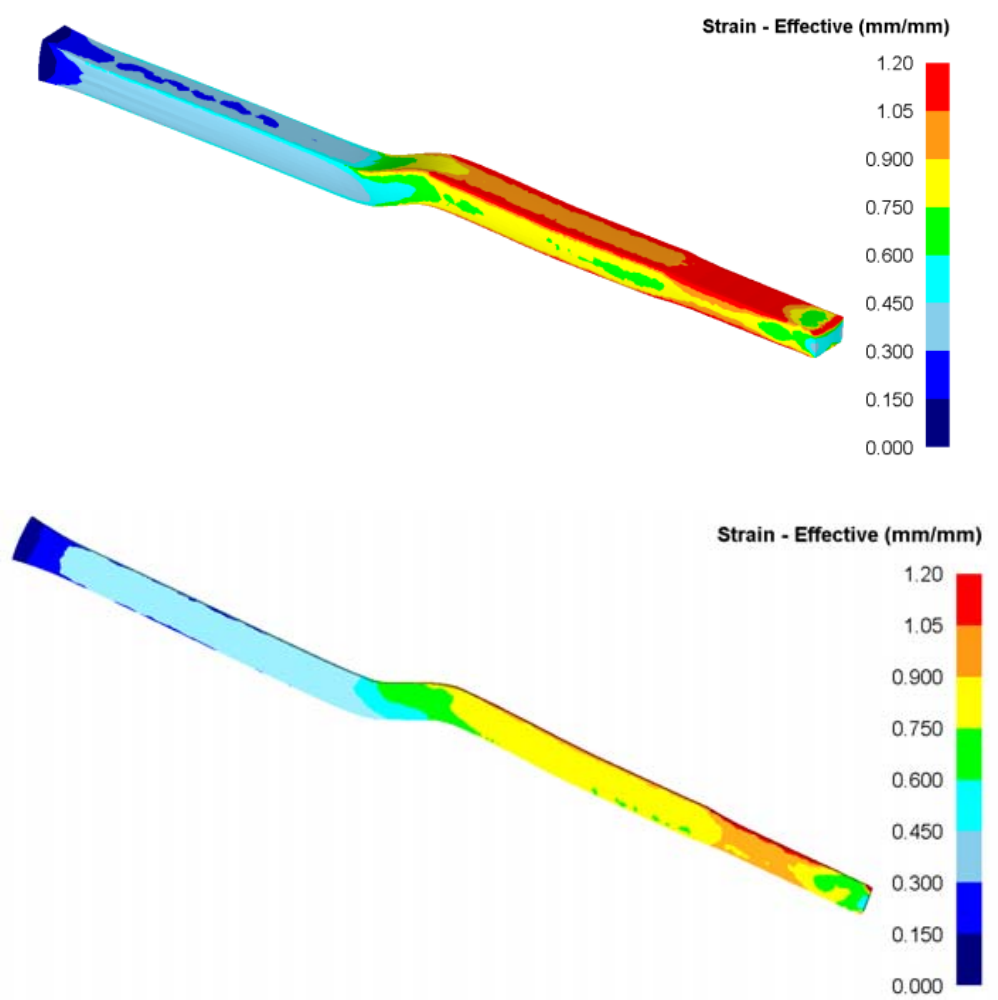

Рис. 2. Эквивалентная деформация

Результаты исследования эквивалентных деформаций в поверхностных и центральных слоях заготовки показали, что распределение этого параметра по сечению неравномерно. Поэтому можно сделать вывод, что при разнице эквивалентных деформаций в центральной зоне до входа в матрицу и после выхода из матрицы, равной 0,6, эта разность представляет собой часть деформации, соответствующую простому сдвигу в матрице. Другими словами, центр заготовки при прохождении каналов матрицы получает увеличение эквивалентной деформации на 0,6 в перпендикулярном направлении к деформации предыдущей фазы и ориентации структуры центральной части. Несколько циклов деформирования для такой схемы должны способствовать уменьшению анизотропии свойств по поперечному сечению стержня.

Далее была поставлена задача - выяснить, как влияет каждый из данных параметров на напряженно-деформированное состояние процесса. С этой целью были построены еще несколько моделей, которые отличались от базовой модели измененным значением лишь одного параметра. Также учитывалась необходимость «реального» варьирования, то есть изменялись только те параметры, которые можно изменить на лабораторно-промышленной установке.

Также необходимо отметить еще один важный технологический параметр, влияющий на возможность осуществления данного совмещенного процесса - расстояние матрицы от очага деформации в валках. В базовой модели данное расстояние было равно 85 мм. В работе [8] рассматривалось влияние данного параметра на возможность реализации данного совмещенного процесса. Было установлено, что это значение должно быть как можно меньше. 
В итоге было принято решение провести варьирование значениями обоих коэффициентов трения, длинами первого канала и величиной угла стыка каналов в матрице. Также было решено провести варьирование температурой нагрева заготовки в оба направления, поскольку для заданной стали 35 исходная величина температуры нагрева $\left(700{ }^{\circ} \mathrm{C}\right)$ является краевым значением рекомендуемого диапазона теплой деформации. ковочных температур $\left(400 \ldots 700^{\circ} \mathrm{C}\right)$.

В результате были построены дополнительные модели со следующими измененными параметрами:

1) с температурами нагрева заготовки $850^{\circ} \mathrm{C}$ и $550{ }^{\circ} \mathrm{C}$;

2) с углом стыка каналов в матрице $160^{\circ}$;

3) с коэффициентом трения в валках 0,7 (что соответствует искусственно созданной поверхности с грубой насечкой);

4) с коэффициентом трения в матрице 0,05 (что соответствует полированной поверхности с применением смазки);

5) с длинами первого канала в матрице 80 мм и 60 мм.

При изучении моделей с протяженностью первого канала в матрице 80 мм и 60 мм было отмечено, что распределение напряжений и деформаций по всему объему заготовки при изменении протяженности первого канала в матрице практически не изменяется. Разница значений параметров НДС составляет не более 5-7 \%. Поэтому снижать длину первого канала в матрице не рекомендуется, так как в этом случае есть риск снижения прочности общей конструкции матрицы.

Поскольку исходная температура нагрева была повышена на $150{ }^{\circ} \mathrm{C}$, то остывание заготовки значительно ниже, чем в базовой модели. При изучении главных компонентов напряжения (как растягивающего, так и сжимающего) было выявлено, что в модели с повышенной температурой нагрева заготовки наблюдается снижение значений во всех очагах деформации. Это связано с тем, что заготовка имеет повышенную температуру и, как следствие, более высокую пластичность, что позволяет ей деформироваться при меньших энергозатратах.

Поскольку эквивалентное напряжение определяется из значений главных напряжений, то в модели с повышенной температурой нагрева заготовки значение этого параметра будет ниже, чем в базовой модели, так как значения главных напряжений снизились из-за повышения температуры заготовки, что привело к повышению уровня пластичности материала. В первом очаге деформации в зонах контакта металла с валками его значение эквивалентного напряжения достигает $75 \mathrm{MПа,} \mathrm{значение} \mathrm{снизилось} \mathrm{на} 28$ \% по сравнению с базовым. На свободных от контакта зонах значение данного параметра находится в пределе 60 МПа, что ниже базового значения на 25 \%. В зоне стыка каналов матрицы величина эквивалентного напряжения достигает 60-65 МПа, значение снизилось на 24 \% по сравнению с базовым. В третьем очаге деформации при прокатке во второй паре валков создается напряжение, достигающее величины 65-70 МПа (ниже базового значения на 14 \%) в центральных слоях заготовки и 80-85 МПа (ниже базового значения на 11 \%) в поверхностных слоях.

При исследовании деформированного состояния в модели с повышенной температурой нагрева заготовки было отмечено общее возрастание уровня эквивалентной деформации. В первом очаге деформации эквивалентная деформация достигает значения 0,47 в поверхностных слоях, что выше базового значения на $17 \%$; в центральных слоях значение данного параметра находится на уровне 0,36, что выше значения в базовой модели на $20 \%$.

Во втором очаге эквивалентная деформация после прохождения первого стыка достигает значения 0,8 в поверхностных слоях, что на $7 \%$ выше базового значения и 0,75 в центральных слоях - это выше на 15 \% по сравнению с базовой моделью. После прохождения второго стыка значение эквивалентной деформации достигает 1,14 в поверхностных слоях, что на $14 \%$ выше базового значения и 1,05 в центральных слоях - это выше на $17 \%$ по сравнению с базовой моделью. В третьем очаге деформации при прокатке во второй паре валков значение эквивалентной деформации достигает 1,3 в поверхностных слоях, что на $8 \%$ выше базового значения и 1,14 в центральных слоях, что выше базового значения на 14 \%. 
Повышение общего уровня деформации является следствием использования заготовки с повышенной температурой нагрева. При данном условии фактическое сопротивление материала значительно ниже. Несмотря на то, что деформация сдвига, по сути, не меняется, так как геометрические параметры процесса остаются неизменными, общий уровень деформации повышается за счет снижения сопротивляемости заготовки пластическому деформированию при прокатке. Возросший уровень эквивалентной деформации, несомненно, носит положительный характер, способствуя более глубокой проработке исходной структуры материала. С другой стороны, повышение температуры нагрева заготовки интенсифицирует процесс статической рекристаллизации, что провоцирует значительный рост зерна, таким образом сводя на нет воздействие деформации.

При изучении эквивалентного напряжения было отмечено, что в модели с пониженной температурой нагрева заготовки значение этого параметра выше, чем в базовой модели, так как значения главных напряжений возросли из-за понижения температуры заготовки, что привело к снижению уровня пластичности материала. В первом очаге деформации в зонах контакта металла с валками его значение эквивалентного напряжения достигает 115 МПа, значение увеличилось на 10 \% по сравнению с базовым. На свободных от контакта зонах значение данного параметра находится в пределе 90 МПа, что выше базового значения на $20 \%$. В зоне стыка каналов матрицы величина эквивалентного напряжения достигает 105 МПа, значение увеличилось на 31 \% по сравнению с базовым. В третьем очаге деформации при прокатке во второй паре валков создается напряжение, достигающее величины 90 МПа (выше базового значения на 28 \%) в центральных слоях заготовки и 115 МПа (выше базового значения на 21 \%)в поверхностных слоях.

При исследовании деформированного состояния в модели с пониженной температурой нагрева заготовки было отмечено снижение уровня эквивалентной деформации. В первом очаге деформации эквивалентная деформация достигает значения 0,3 в поверхностных слоях, что ниже базового значения на 33 \%; в центральных слоях значение данного параметра находится на уровне 0,2 , что ниже значения в базовой модели на $66 \%$.

Во втором очаге эквивалентная деформация после прохождения первого стыка достигает значения 0,6 в поверхностных слоях, что на $20 \%$ ниже базового значения и 0,55 в центральных слоях - это ниже на $16 \%$ по сравнению с базовой моделью. После прохождения второго стыка значение эквивалентной деформации достигает 0,9 в поверхностных слоях, что на 10 \% ниже базового значения и 0,75 в центральных слоях- это ниже на 17 \% по сравнению с базовой моделью. В третьем очаге деформации при прокатке во второй паре валков значение эквивалентной деформации достигает 1,08 в поверхностных слоях, что на 10 \% ниже базового значения и 0,8 в центральных слоях, что ниже базового значения на $20 \%$.

Использование заготовки с пониженной температурой нагрева приводит к понижению общего уровня деформации. В данной модели общий уровень деформации снижается за счет повышения сопротивляемости заготовки пластическому деформированию при прокатке. Снижение уровня эквивалентной деформации в модели с пониженной температурой нагрева заготовки носит нежелательный характер. Однако, понижение температуры нагрева существенно снижает интенсивность процесса статической рекристаллизации, что замедляет рост зерна.

Данная модель, в отличие от двух ранее рассмотренных, имеет измененный геометрический параметр - угол стыка каналов в матрице. При изучении этой модели было выявлено, что параметры НДС, при сравнении с базовой моделью, изменяются лишь в зоне прессования, то есть в очаге деформации матрицы. При этом все исследуемые параметры в очаге прокатных валков практически неизменны (разница значений при сравнении составила 3-5\%, что можно отнести к погрешностям расчета). Поэтому было принято решение проводить изучение данной модели, рассматривая лишь 2-ой этап деформирования.

При рассмотрении эквивалентного напряжения было выявлено снижение данного параметра за счет уменьшения действия подпора. Значение напряжения в зоне стыка каналов матрицы величина эквивалентного напряжения достигает 60-65 МПа, что ниже базового уровня на $20 \%$. 
При изучении эквивалентной деформации было выявлено, что изменение угла стыка каналов оказывает существенное влияние на величину данного параметра. После прохождения первого стыка значение эквивалентной деформации достигает 0,5 в поверхностных слоях, что ниже значения базовой модели на 33 \%, в центральных слоях величина данного параметра находится на уровне 0,45 , что ниже значения базовой модели на 30 \%. После прохождения второго стыка значение эквивалентной деформации достигает 0,65 в поверхностных слоях, что ниже значения базовой модели на 35 \%, в центральных слоях величина данного параметра находится на уровне 0,5 , что ниже значения базовой модели на $45 \%$.

Поскольку изменение значения угла стыка каналов влияет, в основном, на изменение параметров НДС в матрице, то повышение величины угла стыка является нецелесообразным. Несмотря на то, что это приводит к снижению энергозатрат для деформирования, повышение угла стыка каналов негативно сказывается на росте эквивалентной деформации. Особенно критично в этом случае снижение доли деформации простого сдвига, которая должна способствовать преобразованию ориентированной полосчатой структуры центральной части прутка в более равноосную, снижая таким образом анизотропию свойств по всему сечению. В конечном счете, это приведет к необходимости проведения большего количества циклов деформирования, чтобы достичь заданный уровень проработки структуры.

При изучении данных двух моделей были выявлены следующие закономерности:

- изменение параметров напряженного состояния происходит только в том очаге деформации, где непосредственно изменяется коэффициент трения;

- сравнительный анализ значений напряженного состояния показал, что разница изменения параметров минимальна (менее 1 \%). Несмотря на то, что известны результаты исследований зависимости коэффициента трения на параметры НДС различных процессов деформирования [9-10], в которых отмечено существенное влияние данного параметра в данном случае было получено минимальное влияние изменения коэффициента трения в сравнении с базовой моделью. Это объясняется очень малым оптимальным диапазоном варьирования данного параметра, как в валках, так и в матрице.

- изменение параметров деформированного состояния не происходит ни в одном из очагов деформации, что также является следствием малого диапазона варьирования данного параметра.

В результате было принято решение данные модели не рассматривать.

Для изучения эволюции микроструктуры была использована специализированная база данных микроструктуры программы Matilda. Данная программа является инструментом моделирования физико-химических процессов, протекающих в деформируемом теле и является модульной надстройкой к программе Simufact. Matilda использует данные напряженнодеформированного состояния, скорости деформации и температуры из готовой рассчитанной модели в Simufact Forming, дополняет их данными физико-химических свойств и их поведения для заданного материала и его структуры из собственной базы данных, затем, по алгоритму Yada [11], рассчитывает параметры процессов статической и динамической рекристаллизации, которые влияют на изменение размеров зерна. Размер зерна также рассчитывается для каждого узла конечно-элементной модели и отображается в конце моделирования в любой удобной для визуализации форме.

Таким образом, в базовую модель были импортированы данные свойств стали AISI 1035, соответствующей стали 35, из специализированного программного комплекса Matilda. При моделировании микроструктуры в программе используется допущение, что заготовка до деформирования имеет равномерную структуру с одинаковым размером зерна в любой точке. В качестве исходного размера был принят средний диаметр зерна 40 мкм.

После расчета модели были получены результаты эволюции микроструктуры, которые рассматривались отдельно на поверхности и в продольном сечении заготовки. В процессе прокатки поверхностные слои заготовки получают интенсивную деформацию, что способствует 
существенному измельчению зерна от 40 мкм до 30 мкм (рис. 3). После прохождения каналов матрицы за счет реализации сдвиговой деформации зерно дополнительно уменьшается до 22 мкм. После прокатки во второй паре валков размер зерна составляет 18 мкм.

Центральные слои заготовки менее интенсивно обрабатываются при прокатке - здесь размер зерен изменяется от 40 мкм до 32 мкм. После прохождения через каналы матрицы в центральной зоне размер зерна изменяется от 32 мкм до 28 мкм. После прокатки во второй паре валков размер зерна составляет 25 мкм. Таким образом, после одного цикла деформации разница в размерах зерен между поверхностной и центральной зонами составляет $38 \%$. Это подтверждает ранее полученные данные о том, что при осуществлении данного совмещенного процесса заготовка не обрабатывается равномерно по сечению, и для выравнивания свойств по сечению необходимо выполнить несколько циклов деформации.

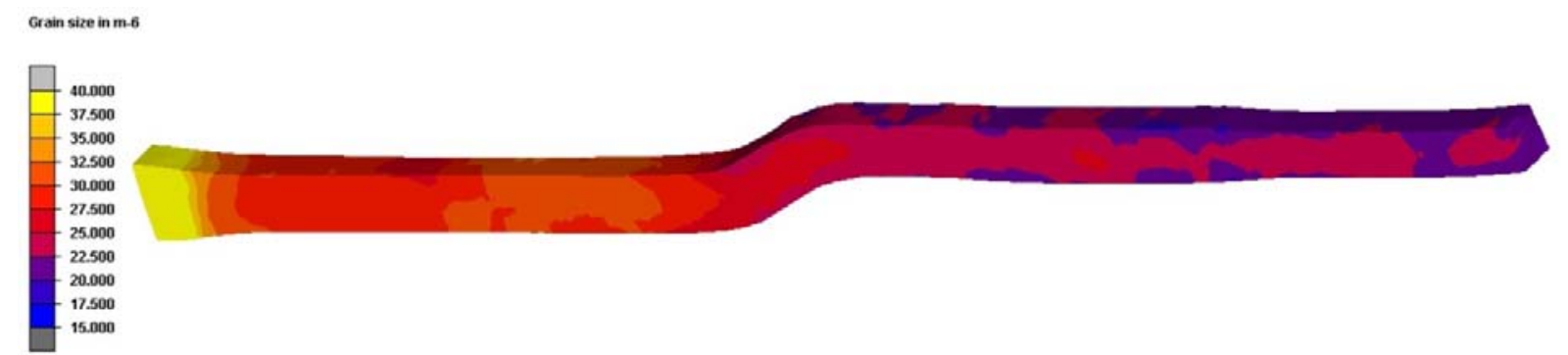

Рис. 3. Изменение размера зерна

После рассмотрения базовой модели была поставлена задача: выявить влияние температуры нагрева заготовки на размер зерна. Этот параметр был выбран из соображений, что он является наиболее гибким с точки зрения изменения значений. Температура довольно легко меняется, когда заготовка нагревается в печи. При изучении влияния температуры на эволюцию микроструктуры использовались ранее построенные модели с температурами предварительного нагрева $850{ }^{\circ} \mathrm{C}$ и $550{ }^{\circ} \mathrm{C}$. Изменение размера зерна было решено рассмотреть только после прокатки во второй паре валков. Изучение этих моделей показало, что влияние температуры на изменение размера зерна очень существенно. Снижение температуры нагрева приводит к интенсификации измельчения исходного зерна, а повышение температуры нагрева значительно замедляет этот процесс. Это связано с тем, что при повышении температуры нагрева металла процессы статической и динамической рекристаллизации, которые нежелательны, значительно ускоряются, так как приводят к появлению и росту новых зерен. При понижении температуры ниже значения начала рекристаллизации процессы рекристаллизации прекращаются, что благоприятно сказывается на интенсивности обработки исходной структуры. При анализе полученных значений было установлено, что в модели с температурой нагрева $850{ }^{\circ} \mathrm{C}$ размер зерна после одного цикла деформации составляет 35 мкм в поверхностной зоне и 38 мкм в центральной зоне. Таким образом, после прохождения через три зоны деформации начальный размер зерна в этой модели изменился только на 5-7 мкм в поверхностной зоне и на 2-4 мкм в центральной зоне. Несмотря на интенсивную обработку металла, столь незначительное изменение начального размера зерна обусловлено процессами статической и динамической рекристаллизации, при которых деформационная обработка практически сводится к нулю.

При анализе модели с температурой нагрева $550{ }^{\circ} \mathrm{C}$ было установлено, что размер зерна после одного цикла деформации составляет 15 мкм в поверхностной зоне и 22 мкм в центральной зоне. Таким образом, после прохождения через три зоны деформации начальный размер зерна в этой модели изменился на 25-28 мкм в поверхностной зоне и на 18-24 мкм в центральной зоне. Столь значительное изменение исходного размера зерна обусловлено отсутствием процессов статической и динамической рекристаллизации, без которых вся деформационная обработка сохраняется в накопленном состоянии. 
Здесь следует отметить, что модели с пониженной температурой нагрева являются оптимальными в плане более интенсивного измельчения зерна. Однако по сравнению с базовыми моделями разница в значениях не является существенной. В то же время снижение пластичности при более низкой температуре приведет к увеличению нагрузок на оборудование. Поэтому, для последующей многопроходной деформации, было решено использовать модели с температурой нагрева $700{ }^{\circ} \mathrm{C}$.

Для многопроходного деформирования была выбрана базовая модель с температурой нагрева заготовки $700{ }^{\circ} \mathrm{C}$, которая была определена как наиболее оптимальная. Деформацию проводили при следующих условиях:

- 1-й проход: заготовку высотой 30 мм прокатывали на 5 мм в первой паре валков, пропускали через матрицу с каналом высотой 26 мм и затем прокатывали на 2 мм во второй паре валков;

- 2-й проход: заготовку высотой 24 мм прокатывали на 3 мм в первой паре валков, пропускали через матрицу с каналом высотой 22 мм и затем прокатывали на 2 мм во второй паре валков;

- 3-й проход: заготовку высотой 20 мм прокатывали на 2 мм в первой паре валков, пропускали через матрицу с каналом высотой 19 мм и затем прокатывали на 2 мм во второй паре валков.

После второго прохода измельчение зерна происходит не так интенсивно, как в первом проходе. Это связано с тем, что уменьшение в этом проходе составило 3 мм, что меньше, чем в первом проходе (5 мм). Здесь средний диаметр зерна в поверхностной зоне составлял 13 мкм, в центральной зоне - 19 мкм, то есть разница составила 6 мкм. После третьего прохода средний диаметр зерна в поверхностной зоне составил 7 мкм, в центральной зоне 11 мкм, то есть разница составила 4 мкм. Таким образом, в результате изучения модели многопроходного деформирования было установлено, что с увеличением числа проходов происходит не только общее уменьшение среднего диаметра зерна, но и постепенное выравнивание этого параметра между центральной и поверхностной зонами.

\section{ВЫВОДЫ}

Для изучения напряженно-деформированного состояния был смоделирован процесс «прокатка-РКУП» с варьированием основных геометрических и технологических факторов, оказывающих существенное влияние на этот процесс. Анализ влияния различных факторов на напряженно-деформированное состояние этого процесса показал, что такие факторы, как угол соединения каналов матрицы и температура нагрева заготовки оказывают существенное влияние на распределение напряжений и накопленных деформаций во всем объеме заготовки в процессе «прокатка-РКУП». При этом вариации коэффициентов трения и длин каналов в матрице в пределах допустимых значений не играют существенной роли в изменении параметров напряженно-деформированного состояния. При изучении эволюции микроструктуры было установлено, что данный комбинированный способ деформирования позволяет значительно измельчить исходный размер зерна. Установлено, что влияние температуры нагрева заготовки играет важную роль в уровне измельчения зерна. Для того чтобы уменьшить разброс значений и выровнять размер зерна по сечению, необходимо подвергнуть заготовку нескольким циклам деформации. Так, после третьего прохода разница в среднем размере зерен между центральной и поверхностной зонами составляет всего 4 мкм, что значительно меньше, чем после первого прохода (7 мкм).

\section{СПИСОК ИСПОЛЬЗОВАННОЙ ЛИТЕРАТУРЫ}

1. Raab G.I., Raab A.G., Zhilyaev A.P. Gradient structure and methods for their preparation. Proceedings of the 16th International Congress «Machines, Technologies, Materials». 2019, Vol. I "Technologies", pp. 132-133.

2. Naizabekov A., Lezhnev S., Panin E., Arbuz A. Combined process "helical rolling-pressing" and its effect on the microstructure of ferrous and non-ferrous materials. Metallurgical Research \& Technology. $2018,115,213$. pp. 11- 19. DOI: https://doi.org/10.1051/metal/2017099 
3. Mochalin I.V., Gorokhov Yu.V., Belyaev S.V., Gubanov I.Yu. Extrusion copper bus bars at the installation "Conform" with prechamber. Nonferrous metals. 2016, 5, pp. 75-78.

4. Pat. 27262 RK. Device for continuous pressing of metals and alloys. Naizabekov A.B., Lezhnev S.N., Panin E.A. 2016.

5. Pat. 25862 RK. Device for continuous pressing of metals and alloys. Naizabekov A.B., Lezhnev S.N., Panin E.A. 2013.

6. Naizabekov A., Lezhnev S., Panin E., Koinov T. Theoretical grounds of the combined "rolling - equal channel step pressing" process. Journal of Chemical Technology and Metallurgy. 2016, 51, 5, pp. 594-602.

7. Naizabekov A., Lezhnev S., Panin E., Volokitina I. New combined technology of deformation "rolling-equal channel angular pressing". In Book: Severe Plastic Deformation Techniques. Ed. M. Cabibbo. 2017. DOI: https://doi.org/10.5772/intechopen.68663

8. Фастыковский А.Р. Оценка возможностей процесса прокатки-прессования. Кузнечно-цтамповочное производство. Обработка металлов давлением. 2004. 2. С. 3-6.

9. Valiev R.Z., Islamgaliev R.K., Alexandrov I.V. Bulk nanostructured materials from severe plastic deformation. Progress in Materials Science. 2000, 45(2), pp. 103-189.

10. Valiev R.Z., Langdon T.G. Principles of equal-channel angular pressing as a processing tool for grain refinement. Progress in Materials Science. 2006, 51, pp. 881-981.

11. Yada H., Matsuzu N., Nakajima K., Watanabe K., Tokita H. Strength and structural-changes under high strain-rate hot deformation of C-steels. Transactions of the Iron and Steel Institute of Japan. 1983, 23, 2, pp. 100-109.

\section{REFERENCES}

1. Raab G.I., Raab A.G., Zhilyaev A.P. Gradient structure and methods for their preparation. Proceedings of the 16th International Congress «Machines, Technologies, Materials». 2019, Vol. I "Technologies", pp. 132-133.

2. Naizabekov A., Lezhnev S., Panin E., Arbuz A. Combined process "helical rolling-pressing" and its effect on the microstructure of ferrous and non-ferrous materials. Metallurgical Research \& Technology. $2018,115,213$. pp. 11- 19. DOI: https://doi.org/10.1051/metal/2017099

3. Mochalin I.V., Gorokhov Yu.V., Belyaev S.V., Gubanov I.Yu. Extrusion copper bus bars at the installation "Conform" with prechamber. Nonferrous metals. 2016, 5, pp. 75-78.

4. Pat. № 27262 RK. Device for continuous pressing of metals and alloys. Naizabekov A.B., Lezhnev S.N., Panin E.A. 2016.

5. Pat. № 25862 RK. Device for continuous pressing of metals and alloys. Naizabekov A.B., Lezhnev S.N., Panin E.A. 2013.

6. Naizabekov A., Lezhnev S., Panin E., Koinov T. Theoretical grounds of the combined "rolling - equal channel step pressing" process. Journal of Chemical Technology and Metallurgy. 2016, 51, 5, pp. 594-602.

7. Naizabekov A., Lezhnev S., Panin E., Volokitina I. New combined technology of deformation "rolling-equal channel angular pressing". In Book: Severe Plastic Deformation Techniques. Ed. M. Cabibbo. 2017. DOI: https://doi.org/10.5772/intechopen.68663

8. Fastikovsky A.R. Evaluation of the capabilities of the rolling process-pressing. Forging and stamping Production. 2004, 2, pp. 3-6. (in Russian).

9. Valiev R.Z., Islamgaliev R.K., Alexandrov I.V. Bulk nanostructured materials from severe plastic deformation. Progress in Materials Science. 2000, 45(2), pp. 103-189.

10. Valiev R.Z., Langdon T.G. Principles of equal-channel angular pressing as a processing tool for grain refinement. Progress in Materials Science. 2006, 51, pp. 881-981.

11. Yada H., Matsuzu N., Nakajima K., Watanabe K., Tokita H. Strength and structural-changes under high strain-rate hot deformation of C-steels. Transactions of the Iron and Steel Institute of Japan. 1983, 23, 2, pp. 100-109.

Найзабеков А. Б. - д-р техн. наук, проф. РИИ;

Лежнев С. Н. - канд. техн. наук, проф. РИИ;

Панин Е. А. $\quad-\mathrm{PhD}$, доцент КГИУ

РИИ - Рудненский индустриальный институт, г. Рудный, Казахстан;

КГИУ - Карагандинский государственный индустриальный университет, г. Темиртау, Казахстан. 